Original Research Article

\title{
Randomized, single blinded comparative study of selective serotonin reuptake inhibitor augmentation with olanzapine v/s amisulpride for obsessive compulsive disorder
}

\author{
O. P. Raichandani ${ }^{1}$, J. N. Chaturvedi ${ }^{2 *}$
}

\begin{abstract}
${ }^{1}$ Department of Psychiatry, Netaji Subhash Chandra Bose Medical College, Jabalpur, Madhya Pradesh, India ${ }^{2}$ Department of Pharmacology, Shyam Shah Medical College, Rewa, Madhya Pradesh, India
\end{abstract}

Received: 03 June 2019

Revised: 29 June 2019

Accepted: 06 July 2019

*Correspondence to:

Dr. J. N. Chaturvedi,

Email: jitendrachaturvedi1986@ gmail.com

Copyright: (C) the author(s), publisher and licensee Medip Academy. This is an openaccess article distributed under the terms of the Creative Commons Attribution NonCommercial License, which permits unrestricted noncommercial use, distribution, and reproduction in any medium, provided the original work is properly cited.

\begin{abstract}
Background: Obsessive-Compulsive Disorder is classified in the Diagnostic and Statistical Manual of Mental Disorders, Fourth Edition, Text Revision (DSM-IVTR) as an anxiety disorder. Serotonin reuptake inhibitors are considered to be most effective and are the first line pharmacotherapy for the treatment of OCD. However, about $40-60 \%$ of OCD patients fail to respond to SSRI mono-therapy. Further, as many as $25 \%$ of patients fails to experience any improvement from initial SSRIs mono-therapy. For non-responder's low dose augmentation with antipsychotics (risperidone, quetiapine, olanzapine, aripirazole, amisuplride etc.) has shown promising response, as compared to serotonin enhancers. The present study is designed to evaluate and compare the efficacy and adverse drug reactions of these two antipsychotics viz. Olanzapine and amisulpride as augmentation strategy in OCD patients. Objective of present study was to compare the efficacy of olanzapine and amisulpride as add on therapy for inadequately controlled obsessive-compulsive disorder patients on selective-serotonin reuptake inhibitor. Methods: the present study was done at Medical College Jabalpur (M.P.) in the department of Psychiatry \& Pharmacology. It was randomized, patient blinded study. 47 patients were screened for the study out of which 36 were enrolled and randomized into either SSRI+Olanzapine or SSRI+Amisulpride group. The patients were evaluated at baseline and then biweekly for 12 weeks to assess the efficacy of these drugs as augmentation strategy using Yale-Brown Obsessive Compulsive Scale (Y-BOCS).

Results: There was a significant improvement in Yale-Brown Obsessive Compulsive Scale (Y-BOCS), Clinical Global Impression-Severity (CGI-S) score and Clinical Global impression-Improvement (CGI-I) score in both the groups but there was no significant difference $(\mathrm{P}>0.05)$ in either of these groups on these three scale. No serious adverse drug reaction was reported in either of these groups.
\end{abstract}

Conclusions: Both olanzapine and amisulpride are efficacious and well tolerated for augmentation of SSRI with no significant difference in their efficacy.

Keywords: Amisulpride, Augmentation, Obsessive Compulsive Disorder, Olanzapine, Selective Serotonin Reuptake Inhibitors

\section{INTRODUCTION}

Obsessive-Compulsive Disorder is classified in the Diagnostic and Statistical Manual of Mental Disorders, Fourth Edition, Text Revision (DSM-IV-TR) as an anxiety disorder. The disorder is characterized by either obsession or compulsion or both. Obsession is defined as recurrent and persistent thoughts, impulses, or images that are experienced at some time during the disturbance, as intrusive and inappropriate and that cause marked anxiety 
or distress. Compulsion is a repetitive behaviours (e.g., hand washing, ordering, checking) or mental acts (e.g., praying, counting, repeating words silently) that the person feels driven to perform in response to an obsession. ${ }^{1}$

OCD is the fourth common psychiatric disorder and tenth common cause of disability worldwide. Total burden of the disease (as measured by DALY-Disability Adjusted Life years) is $2.2 \%$ of the total causes, making it tenth most common disability. ${ }^{2}$

The epidemiological studies in India have estimated the lifetime prevalence of $0.6 \% .^{3}$ This rate is considerably lower compared to the 2-3\% rate reported in the European and North American studies. ${ }^{4}$ However, further research is needed into the epidemiological aspects of OCD in India since the data available is limited.

The average age of onset for OCD varies among men and women. Men may experience childhood onset, between ages 6 and 15, while women typically experience adult onset, between ages 20 and 30. Two-thirds of all adults with OCD had symptoms before age $15,80 \%$ of whom also had symptoms of depression. Given the likelihood of early onset, it is alarming that the average age that a person seeks treatment is $27 .^{5}$

Pharmacotherapy for OCD dates back to mid-1980s when the clomipramine, only tricyclic antidepressant approved for OCD and SRIs were introduced. ${ }^{6}$ Prior to these pharmacological modalities, OCD was considered to be one of the poorly treated psychiatric disorders with chronic and unremitting course. ${ }^{7}$ After the introduction of SRIs certainly there is improved outcome in many patients with OCD. ${ }^{8}$

Serotonin reuptake inhibitors are considered to be most effective and are the first line pharmacotherapy for the treatment of OCD. ${ }^{9}$ However, about $40-60 \%$ of OCD patients fail to respond to SSRI mono-therapy. ${ }^{10,11}$ Further, as many as $25 \%$ of patients fails to experience any improvement from initial SSRIs mono-therapy. ${ }^{12}$

For non-responders to mono-therapy some other pharmacotherapeutic approach are used. Firstly, switching them to other potent SSRI, if not given, and results from this approach showed approximately 33\% response. $^{9}$ Second approach is the augmentation strategy; augmentation with serotonin enhancers (Lithium, clonazepam, and buspirone) can be done. But these agents have yielded uniformly discouraging results. ${ }^{13,14}$ On the other hand, low dose augmentation with antipsychotics (risperidone, quetiapine, olanzapine, aripirazole, amisuplride etc.) has shown promising response, as compared to serotonin enhancers. ${ }^{15}$

Systematic meta-analysis has shown that, only one-third of treatment refractory OCD patients exhibit a meaningful treatment response to antipsychotic medications. ${ }^{16}$
As far as etiology is concerned, there are evidences for the involvement of corticobasal ganglia-thalamo-cortical network in the pathogenesis of OCD. ${ }^{17}$ This network is innervated by serotonergic and dopaminergic neurons. So, from neurotransmitter point of view, dopamine excess and serotonin deficit plays an important role in the causation of OCD. ${ }^{18}$

Olanzapine is an atypical antipsychotic agent. The olanzapine formulations were manufactured and marketed by the Eli Lilly and Company; the drug went generic in 2011. It was approved by FDA for treatment of schizophrenia and bipolar disorder. It is also used as augmentation agent for the management of OCD. ${ }^{19}$ The pharmacology of olanzapine states that it has antagonistic/ inverse agonistic activity at various serotonergic, dopaminergic, muscarinic receptors etc. Affinity for $5 \mathrm{HT} 2 \mathrm{~A}>5 \mathrm{HT} 2 \mathrm{C}=\mathrm{D} 2$ receptor. ${ }^{20}$

Amisulpride is also an atypical antipsychotic it was introduced in the 1990s, used for treatment of psychosis in schizophrenia and episodes of mania in bipolar disorder. Its pharmacology is receptor antagonism on D2 D3 receptors. ${ }^{21}$ It also has $5 \mathrm{HT}_{7}$ antagonistic activity similar to risperidone and ziprasidone. Only one positive study is there with this agent as augmentation strategy in OCD. ${ }^{22}$

The present study is designed to evaluate and compare the efficacy and adverse drug reactions of these two antipsychotics viz. Olanzapine and amisulpride as augmentation strategy in OCD patients.

Objectives is to study the efficacy of Olanzapine and Amisulpride as augmentation therapy in OCD patients not adequately controlled by SSRI monotherapy and to compare the efficacy of Olanzapine and Amisulpride as augmentation therapy for OCD.

\section{METHODS}

\section{Study area}

After getting approval from the institutional Human ethics committee, the study was conducted in the department of Psychiatry \& Pharmacology, Govt. Medical College Jabalpur (M.P.) between the periods from October 2011 to September 2012. Written informed consent was taken from all the patients enrolled in the study and principles of Good Clinical Practice were followed throughout the study period.

\section{Inclusion criteria}

- $\quad$ Patients of either gender \& age between 18-60 years suffering from Obsessive-Compulsive Disorder as per DSM-IV criteria.

- Patient on single SSRI for >3 months and dose escalation has been already done to maximum for that SSRI. 
- The Yale-Brown Obsessive Compulsive Scale (YBOCS) score is $>$ or $=24$.

\section{Exclusion criteria}

- Presence of psychotic disorder/ suicidal tendency/ substance abuse.

- Patients requiring ECT/Hospitalisation/Behavioural therapy.

- Abnormal lipid profile, ECG, blood glucose level or BMI.

- Pregnant women/ women not using contraceptives/ women desiring to have children/ Nursing mothers.

- Patients with hypertension/Cardiac Disorder/ Active liver disease.

\section{Study design}

This study is an OPD based convenient sampling, randomized, patient blinded comparative study. 47 patients attending the psychiatric OPD were screened using these criteria and out of which 36 were enrolled in the study.

The randomisation was done by a biostatistician, using random number table. The randomisation concealment was done by making two copies of the random sequence and sealed in opaque envelop. One envelop was given to the consulting psychiatrist and other was kept with the biostatistician. It was also maintained in electronic database of the statistician. Patient blinding was maintained by giving the drug to the patient in white boxes labelled with either A1-4 or B1-4.

\section{Dose admistration}

For amisulpride the starting dose was $100 \mathrm{mg}$ /day added to the ongoing SSRI treatment and can be titrated up to $400 \mathrm{mg}$ /day in flexible doses.

For olanzapine starting dose was $2.5 \mathrm{mg} /$ day and can be titrated up to $10 \mathrm{mg} /$ day in flexible doses.

Compliance was checked by inquiry from the relatives and pill count by the psychiatrist.

\section{Clinical evaluation}

Baseline Y-BOCS score was noted (at Day 0). The symptom changes using Y-BOCS was evaluated at day 15 , day 30, day 45, day 60 and day 90 .

Severity of the illness was evaluated simultaneously by the psychiatrist on CGI-Severity scale at each visit i.e., Day 0, Day 15, Day 30, Day 45, Day 60 and Day 90.

During this evaluation period, if the psychiatrist feels to change the dose then this was done as per CGIImprovement. The dose was increased in either group if there was no change in CGI-Improvement at Day 30 by 1 point or more from the previous evaluation. And then subsequently on each visit, at the interval of 15 days the dose was adjusted as per the same criteria. The dose was kept same for those showing the improvement by 1 point or more at each evaluation time.

\section{Tools of the study}

The Yale-Brown Obsessive Compulsive Scale (Y-BOCS) was designed to remedy the problems of existing rating scales by providing a specific measure of the severity of symptoms of obsessive-compulsive disorder that is not influenced by the type of obsessions or compulsions present. The scale is a clinician-rated, 10-item scale. Item 1-5 inquiries about the severity of obsession. Item 6-10 inquiries about the compulsion. Each item rated from 0 (no symptoms) to 4 (extreme symptoms). Y-BOCS ranges from $0-40$.

Clinical global impression (CGI) is a clinical evaluation of the syndrome under assessment. The CGI measures overall illness severity and response to treatment. There are seven categories of severity ranging from " $1=$ not ill" to "7=extremely ill". Improvement is also rated on seven point spectrum " $1=$ very much improved" to " $7=$ very much worse" and " $0=$ not evaluated". The CGI is simple, very sensitive and based on clinical experience.

\section{data analysis}

The data of the present study were recorded into the computer and after proper validation, check for error, coding and decoding were compiled and analysed using the software SPSS 19 for windows. At the end of study 31 patients were evaluated, 15 in SSRI+amisulpride group and 16 in SSRI+olanzapine group. Scores like Y-BOCS, CGI-S, CGI-I were expressed as (mean \pm SD) and median (Range).

Unpaired t-test was applied to the two independent groups i.e. amisulpride+SSRI and olanzapine+SSRI for Y-BOCS, CGI-S, CGI-I scores.

For Y-BOCS score, clinically meaningful value was an improvement of the score by $>35 \%$. For CGI, the meaningful response was $\geq 2$ point improvement.

\section{RESULTS}

Both the groups tolerated the treatment and out of 36 patients that were enrolled at the beginning of the study, 31 were evaluated at the end of study. Majority of the patients were in the age group of 20-40 years, 14/18 (77.8\%) in SSRI+ amisulpride group and 16/18 (88.9\%) in SSRI+olanzapine group. Male to female ratio in SSRI+amisulpride group was 1.25:1. While in SSRI+olanzapine group $\mathrm{M}: \mathrm{F}=1.57: 1$. Overall $\mathrm{M}: \mathrm{F}$ ratio in this study is $1.47: 1$. The mean duration of illness in SSRI+Amisulpride group is 10.44 (7.11) years, while 
mean duration of illness in SSRI+Olanzapine group is 10.33 (7.53) years. Dropouts in the SSRI+Amisulpride group were $3 / 18(16.67 \%)$, while $2 / 18(11.11 \%)$ subjects were dropped in the SSRI+Olanzapine group. Overall dropout rate was $5 / 36(13.89 \%)$. Table 1 shows that SSRI+amisulpride group mean Y-BOCS score at day ' 0 ' was reduced from 29.39 (4.34) to 16.27 (4.46) at day ' 90 '. While in SSRI+olanzapine group mean Y-BOCS score was reduced from 29.61(3.40) to 16.63 (3.34) at day ' 90 '. The difference of mean at each point of observation was statistically insignificant on independent $t$-test $(\mathrm{P}>0.05)$.
Table 2 shows that, in SSRI+amisulpride group 10/15 (66.67\%) study subjects has shown clinically meaningful response while in SSRI+olanzapine group 10/16 (62.50\%) has shown clinically meaningful response at the end of study (improvement in Y-BOCS score by $>35 \%$ ). Table 3 shows that, in SSRI+amisulpride group mean CGISeverity score at Day '0' was reduced from $5.44(0.70)$ to 3.73 (0.79) at day '90'. While in SSRI+olanzapine group mean CGI-Severity score at Day ' 0 ' was reduced from $5.39(0.61)$ to $3.81(0.75)$ at day ' 90 '.

Table 1: Comparison of Efficacy by using Y-BOCS Score.

\begin{tabular}{|lllll|}
\hline \multirow{2}{*}{ Day } & \multicolumn{2}{l}{ SSRI + Amisulpride Group } & \multicolumn{2}{l|}{ SSRI + Olanzapine Group } \\
\hline 0 & No. of patients & Mean Score (SD) & No. of Patients & Mean Score (SD) \\
\hline 15 & 18 & $29.39(4.34)$ & 18 & $29.61(3.40)$ \\
\hline 30 & 18 & $29.17(4.27)$ & 17 & $29.53(2.76)$ \\
\hline 45 & 17 & $25.70(4.33)$ & 16 & $26.81(2.32)$ \\
\hline 60 & 16 & $18.69(5.21)$ & 16 & $19.43(3.60)$ \\
\hline 90 & 16 & $16.69(5.08)$ & 16 & $16.93(3.66)$ \\
\hline
\end{tabular}

Table 2: Comparison of percentage improvement in Y-BOCS score from baseline score.

\begin{tabular}{|lll|}
\hline \% improvement & No. of Patients in SSRI + Amisulpride group & No. of Patients in SSRI + Olanzapine group \\
\hline$>35$ & 10 & 10 \\
\hline $25-35$ & 5 & 4 \\
\hline$<25$ & 0 & 2 \\
\hline
\end{tabular}

Table 3: Comparison on CGI-severity score.

\begin{tabular}{|lllll|}
\hline \multirow{2}{*}{ Day } & \multicolumn{2}{l}{ SSRI + Amisulpride Group } & \multicolumn{2}{l|}{ SSRI + Olanzapine Group } \\
\hline 0 & No. of Patients & Mean Score (SD) & No. of Patients & Mean Score (SD) \\
\hline 15 & 18 & $5.44(0.70)$ & 18 & $5.39(0.61)$ \\
\hline 30 & 18 & $5.39(0.69)$ & 17 & $5.12(0.33)$ \\
\hline 45 & 17 & $4.82(0.53)$ & 16 & $4.87(0.34)$ \\
\hline 60 & 16 & $4.06(0.85)$ & 16 & $4.50(0.63)$ \\
\hline 90 & 16 & $3.81(0.83)$ & 16 & $3.94(0.77)$ \\
\hline
\end{tabular}

Table 4: Comparison of changes in CGI-Severity score from baseline.

\begin{tabular}{|lll|}
\hline Improvement & No. of patients in SSRI + Amisulpride group & No. of Patients in SSRI + Olanzapine group \\
\hline$>2$ Points & 2 & 3 \\
\hline 2 Points & 6 & 3 \\
\hline$<2$ Points & 5 & 10 \\
\hline
\end{tabular}

Table 5: Comparison on CGI-Improvement Score.

\begin{tabular}{|lllll|}
\hline \multirow{2}{*}{ Day } & \multicolumn{2}{l|}{ SSRI + Amisulpride Group } & \multicolumn{2}{l|}{ SSRI + Olanzapine Group } \\
\hline 15 & No. of Patients & Mean Score (SD) & No. of Patients & Mean Score (SD) \\
\hline 30 & 18 & $4.00(0.34)$ & 17 & $4.00(0.50)$ \\
\hline 45 & 17 & $3.47(0.79)$ & 16 & $3.69(0.48)$ \\
\hline 60 & 16 & $2.75(0.58)$ & 16 & $2.62(0.96)$ \\
\hline 90 & 16 & $2.37(0.62)$ & 16 & $2.37(0.81)$ \\
\hline
\end{tabular}


Table 6: Comparison of changes in CGI-Improvement score from baseline.

\begin{tabular}{|lll|}
\hline Improvement & No. of patients in SSRI + Amisulpride group & No. of Patients in SSRI + Olanzapine group \\
\hline$>2$ Points & 1 & 2 \\
\hline 2 Points & 9 & 9 \\
\hline$<2$ Points & 5 & 5 \\
\hline
\end{tabular}

Table 7: Comparison of mean dose of Amisulpride and Olanzapine (Post Hoc).

\begin{tabular}{|lll|}
\hline Day & A misulpride Dose in mg (SD) & Olanzapine Dose in mg (SD) \\
\hline 30 & $147.06(51.45)$ & $4.06(1.22)$ \\
\hline 45 & $206.25(44.25)$ & $4.84(1.9)$ \\
\hline 60 & $262.50(50)$ & $6.41(2.05)$ \\
\hline 90 & $273.30(59.4)$ & $6.40(1.8)$ \\
\hline
\end{tabular}

The difference of mean at each point of observation was statistically insignificant on independent t-test $(\mathrm{P}>0.05)$. Table 4 shows that, in SSRI+amisulpride group 8/15 $(53.33 \%)$ of the study subjects had shown clinically meaningful response while in SSRI+olanzapine group $6 / 16(37.50 \%)$ of the study subjects had shown clinically meaningful response at the end of study $(\geq 2$ point improvement).

Table 5 shows that, in SSRI+amisulpride group baseline mean CGI-Improvement score of 18 subjects at Day ' 15 ', was $4.00(0.34)$ with a median of 4.00 (3-5). 15 subjects completed the study and were evaluated for improvement at Day '90', mean CGI-Improvement at the end of the study was 2.20 (0.56) with a median score of 2 (1-3). In SSRI+olanzapine group Baseline mean CGI-Improvement of 17 subjects at Day ' 15 ' was $4.00(0.50)$ with a median of 4.00 (3-5). 16 subjects completed the study and were evaluated for improvement at Day '90', mean CGIImprovement at the end of the study was $2.18(0.65)$ with a median score of 2 (1-3). The difference of mean at each point of observation was statistically insignificant on independent t-test $(\mathrm{P}>0.05)$. Table 6 shows that in SSRI+amisulpride group $10 / 15(66.67 \%)$ of the study subjects had shown clinically meaningful response while in SSRI+olanzapine group $11 / 16(68.75 \%)$ of the study subjects had shown clinically meaningful response $(\geq 2$ point improvement). Table 7 shows that mean dose of amisulpride at the end of Day '90' is 273.30 (59.4) mg and that of olanzapine at day '90' was $6.40(1.8) \mathrm{mg}$.

\section{DISCUSSION}

In this randomized double blind study initially 47 patients of Obsessive-Compulsive disorder on single SSRI were screened and on the basis of laboratory investigations, inclusion and exclusion criteria 36 patients were enrolled in the study. They were randomized into either Amisulpride group or Olanzapine group on the basis of random sequence generated by block randomization, 18 in each group.
Final analysis was done in 15 patients in SSRI+Amisulpride group and in 16 patients in SSRI+Olanzapine group. Hence, the overall dropout rate for this study was 5/36 (13.89\%). In Amisulpride group the initial dose was $100 \mathrm{mg} / \mathrm{d}$ and in Olanzapine group it was $2.5 \mathrm{mg} / \mathrm{d}$. Mean dose of amisulpride was $273.3 \pm 59.4 \mathrm{mg} / \mathrm{d}$ and for Olanzapine mean dose was $6.4 \pm 1.8 \mathrm{mg} / \mathrm{d}$. O. Metin et al, studied amisulpride augmentation and their study found the clinical response with a mean dose of $325 \pm 106$ $\mathrm{mg} / \mathrm{d}$. Shapira et al, in their double blind placebo controlled olanzapine augmentation study had the mean dose of $6.1 \pm 2.1 \mathrm{mg} / \mathrm{d}$ but they did not found significant improvement with this dose. A Bystritsky et al, in their double blind placebo controlled study with olanzapine found significant improvement with mean dose of $11.2 \pm 6.5 \mathrm{mg} / \mathrm{d}^{22-24}$

The patients were evaluated on Y-BOCS and CGI at the interval of 15 days for a period of 12 weeks. We also used the criteria of $>35 \%$ improvement on Y-BOCS and $\geq 2$ point improvement to be clinically meaningful. The mean change on Y-BOCS score in SSRI+Amisulpride was from 29.39 (4.34) to 16.27 (4.46) and in SSRI+Olanzapine group was from 29.61 (3.40) to 16.63 (3.34). Clinically meaningful response on Y-BOCS was seen in 10/15 $(66.67 \%)$ patients in SSRI+Amisulpride group and in $10 / 16(62.50 \%)$ in SSRI+Olanzapine group. On CGISeverity scale the change in SSRI+Amisulpride group was from $5.44(0.70)$ to $3.73(0.79)$ and in SSRI+Olanzapine group the change was from $5.39(0.61)$ to $3.81(0.75)$. Clinically meaningful response on CGI-S was seen in $8 / 15$ $(53.33 \%)$ in SSRI+Amisulpride group and 6/16 (37.50\%) in SSRI+olanzapine group. On CGI-Improvement scale the change in SSRI+Amisulpride group was from 4.00 (0.34) to $2.20(0.56)$ and in SSRI+Olanzapine group the change was from $4.00(0.50)$ to $2.18(0.65)$. Clinically meaningful response on CGI-I was seen in 10/15 (66.67\%) in SSRI+Amisulpride group and 11/16 (68.75\%) in SSRI+olanzapine group. Overall patients responded significantly in both the groups but the difference in both groups was insignificant. 
In the study of $\mathrm{O}$. Metin et al. with amisulpride, the reduction in Y-BOCS scores was from 26.7 (6.3) to 12.5 (2.8). They used the response criteria as $\geq 60 \%$ reduction in Y-BOCS score as 'significant improvement', 30\%-60\% as 'partial improvement' and $<30 \%$ reduction as 'no improvement'. On their criteria $7 / 20$ (35\%) patients showed significant improvement, $12 / 20(60 \%)$ patients showed partial improvement and $1 / 20(5 \%)$ patients showed no improvement. ${ }^{22}$

Weiss EL et al, reported the case series of SSRI augmentation with olanzapine of 10 patients. Out of which 9 completed the study and the result of the study was, 4/9 $(44.4 \%)$ demonstrated a complete remission or major improvement in obsessive-compulsive symptoms, 3/9 $(33.3 \%)$ had partial remission, and $2 / 9$ (22.2\%) experienced no benefit. ${ }^{25}$ Francobandiera $\mathrm{G}$, also reported a case series of 9 patients with olanzapine. 6/8 (75.0\%) of patients had shown improvement on Y-BOCS and CGI score. $2 / 8(25.0 \%)$ of patients did not respond at all. ${ }^{26}$

Maina $\mathrm{G}$ et al, compared the efficacy of olanzapine and risperidone. Ninety-six subjects with DSM-IV OCD (YBOCS $>$ or $=16$ ) entered the open-label prospective phase; at the end of the 16-week period, 50 (52\%) were judged to be resistant and were randomized to receive risperidone ( 1 to $3 \mathrm{mg} / \mathrm{d})$ or olanzapine $(2.5$ to $10 \mathrm{mg} / \mathrm{d}$ ) addition for 8 weeks. Overall, patients in both groups responded significantly, without differences between the two treatment groups. ${ }^{27}$

D'Amico $G$ et al, studied the effect of adjunctive olanzapine in patients with obsessive-compulsive disorder (OCD) refractory to paroxetine. ${ }^{28}$ Twenty-one patients unresponsive to treatment with paroxetine, administered for at least 12 weeks at the dose of $60 \mathrm{mg} /$ day, participated to a 12-week open-label, add-on trial with olanzapine (10 $\mathrm{mg} /$ day). The psychopathological state was evaluated by the Yale-Brown Obsessive-Compulsive Scale (Y-BOCS) and by Clinical Global Impression (CGI). Three patients did not complete the 12-week adjunctive treatment with olanzapine. In the 18 completers, the mean Y-BOCS score decreased significantly from $27.1 \pm 4.0$ at baseline to $20.1 \pm 3.9$ at final evaluation $(\mathrm{P}<0.001)$. Seven patients $(38.9 \%)$ were rated as responders at final evaluation.

Bystritsky A et al, studied the efficacy of adding an atypical antipsychotic, olanzapine, to a serotonin reuptake inhibitor (SRI) in treatment-refractory obsessivecompulsive disorder (OCD) ${ }^{24}$ Twenty-six patients aged between 18 and 65 (mean $=41.2, \mathrm{SD}=11.9)$ years meeting DSM-IV criteria for OCD, who had not responded to SRIs, were treated for 6 weeks in a double-blind, placebocontrolled augmentation study with either olanzapine (up to $20 \mathrm{mg}$ /day) or placebo. Severity of illness was assessed biweekly by the Yale-Brown Obsessive Compulsive Scale (Y-BOCS). Analysis of covariance with baseline Y-BOCS score included as a covariate was used to compare improvement in Y-BOCS scores in the 2 groups. Response was defined as a $25 \%$ or greater improvement in $\mathrm{Y}$-BOCS score. Six $(46 \%)$ of 13 subjects in the olanzapine group showed a $25 \%$ or greater improvement in Y-BOCS score compared with none in the placebo group.

Shapira et al, did a double-blind, placebo-controlled trial of olanzapine addition in fluoxetine-refractory obsessivecompulsive disorder. ${ }^{23}$ In their study, both the fluoxetineplus-olanzapine $(n=22)$ and fluoxetine-plus-placebo $(\mathrm{n}=22)$ groups improved significantly over 6 weeks $[\mathrm{F}(3,113)=11.64, \mathrm{p}<0.0001]$ according to Yale-Brown Obsessive Compulsive Scale scores with repeatedmeasures analysis of variance. Their findings indicate no additional advantage of adding olanzapine for 6 weeks in OCD patients who have not had a satisfactory response to fluoxetine for 8 weeks, compared with extending the monotherapy trial.

\section{CONCLUSION}

In conclusion, this study suggests that, augmentation with amisulpride as well as olanzapine is efficacious and well tolerated in patients with refractory ObsessiveCompulsive disorder. The difference in efficacy between amisulpride and olanzapine as augmenting agents is not significant.

\section{Funding: No funding sources}

Conflict of interest: None declared

Ethical approval: The study was approved by the Institutional Ethics Committee

\section{REFERENCES}

1. American Psychiatric Association Diagnostic and Statistical Manual of Mental Disorders. 4th Edition, Text Revision (DSM-IV-TR). Washington, DC; 2000.

2. Murray CJL, Lopez AD, eds. Global burden of disease: a comprehensive assessment of mortality and disability from diseases, injuries and risk factors in 1990 and projected to 2020. Vol 1. Boston: Harvard Press; 1996.

3. Khanna S, Gururaj G, Sriram TG. Epidemiology of obsessive-compulsive disorder in India. Presented at: The first International Obsessive-Compulsive Disorder Congress; 1993 March;9-12; Capri.

4. Hollander E, Greenwald S, Neville D, Johnson J, Hornig CD, Weissman MM. Uncomplicated and comorbid obsessive-compulsive disorder in an epidemiologic sample. CNS Spectrums. 1998 May;3(S1):10-8.

5. NIMH Anxiety Disorder Publication [Internet]. Updated April 2008. Available at: https://www.nimh.nih.gov/health/topics/obsessivecompulsive-disorder ocd/index.shtml. Accessed 24 September 2011.

6. Clomipramine Collaborative Study Group. Clomipramine in the treatment of patients with obsessive-compulsive disorder. Arch Gen Psychiatry. $1991 ; 48: 730-8$. 
7. Skoog G, Skoog I. A 40-year follow-up of patients with obsessive-compulsive disorder. Arch Gen Psychiatry. 1999 Feb 1;56(2):121-7.

8. Berrios GE, Chiu H. Obsessive-compulsive disorders in Cambridgeshire: a follow-up study of up to 20 years. Brit J Psychiatry. 1989 May;154(S4):17-20.

9. Expert Consensus Panel for Obsessive-Compulsive Disorder. Treatment of obsessive-compulsive disorder. J Clin Psychiatry. 1997;58(suppl 4): 2-72.

10. Erzegovesi S, Cavallini MC, Cavedini P, Diaferia G, Locatelli M, Bellodi L. Clinical predictors of drug response in obsessive-compulsive disorder. J Clin Psychopharmacol. 2001 Oct 1;21(5):488-92.

11. Ravizza L, Barzega G, Bellino S, Bogetto F, Maina G. Predictors of drug treatment response in obsessivecompulsive disorder. J Clin Psychiatry. 1995;56(8):368-73.

12. Wheadon DE, Bushnell WD, Steiner M. A fixed-dose comparison of 20,40 or $60 \mathrm{mg}$ paroxetine or placebo in the treatment of obsessive-compulsive disorder. Presented at the $32^{\text {nd }}$ Annu Meeting Am Col Neuropsychopharmacol. 1993 Dec;13-17.

13. Mcdougle CJ, Price LH, Goodman WK, Charney DS, Heninger GR. A controlled trial of lithium augmentation in fluvoxamine-refractory obsessivecompulsive disorder: lack of efficacy. J Clin Psychopharmacol. 1991 Jun;11(3):175-84.

14. Pigott TA, L'Heureux F, Hill JL, Bihari K, Bernstein SE, Murphy DL. A double-blind study of adjuvant buspirone hydrochloride in clomipramine-treated patients with obsessive-compulsive disorder. Journal of clinical psychopharmacology. J Clin Psychopharmacol. 1992 Feb;12(1): 11-18.

15. McDougle CJ, Goodman WK, Price LH, Delgado PL, Krystal JH, Charney DS et al. Neuroleptic addition in fluvoxamine-refractory obsessive compulsive disorder. Am J Psychiatry 1990;147(5):652-654.

16. Bloch MH, Landeros-Weisenberger A, Kelmendi B, Coric V, Bracken MB, Leckman JF. A systematic review : Antipsychotic augmentation with treatment refractory Obsessive-Compulsive Disorder. Mol Psychiatry. 2006;11(7):622-32.

17. Insel TR. Toward a neuroanatomy of obsessivecompulsive disorder. Arch Gen Psychiatry. 1992 Sep 1;49(9):739-44.

18. Goodman WK, McDougle CJ, Price LH, et al. Beyond the serotonin hypothesis: a role for dopamine in some forms of obsessive compulsive disorder? J Clin Psychiatry. 1990;51(Suppl):36-43.

19. Olanzapine Prescribing Information. [Internet]. 1997 [Updated 2009 March 19; cited 2011 Sep. 15]. Available at: https://www.accessdata.fda.gov/drugsatfda_docs/labe 1/2009/020592s051,021086s030,021253s036lbl.pdf.

20. Bymaster FP, Calligaro DO, Falcone JF, Marsh RD, Moore NA, Tye NC, Seeman P, Wong DT.
Radioreceptor binding profile of the atypical antipsychotic olanzapine. Neuropsychopharmacology. 1996 Feb;14(2):87-96.

21. Rosenzweig P, Canal M, Patat A, Bergougnan L, Zieleniuk I, Bianchetti G. A review of the pharmacokinetics, tolerability and pharmacodynamics of amisulpride in healthy volunteers. Human Psychopharmacology: Clinical and Experimental. 2002 Jan;17(1):1-3.

22. Metin Ö, Yazici K, Tot S, Yazici AE. Amisulpiride augmentation in treatment resistant obsessivecompulsive disorder: an open trial. Human Psychopharmacology: Clinical and Experimental. 2003 Aug;18(6):463-7.

23. Shapira NA, Ward HE, Mandoki M, Murphy TK, Yang MC, Blier P, Goodman WK. A double-blind, placebo-controlled trial of olanzapine addition in fluoxetine-refractory obsessive-compulsive disorder. Biol Psychiatry. 2004 Mar 1;55(5):553-5.

24. Bystritsky A, Ackerman DL, Rosen RM, Vapnik T, Gorbis E, Maidment KM, et al. Augmentation of serotonin reuptake inhibitors in refractory obsessivecompulsive disorder using adjunctive olanzapine: a placebo-controlled trial. J Clin Psychiatry. 2004 Apr;65:565-8.

25. Weiss EL, Potenza MN, McDougle CJ, Epperson CN. Olanzapine addition in obsessive-compulsive disorder refractory to selective serotonin reuptake inhibitors: an open-label case series. J Clin Psychiatry. 1999 Aug;60(8):524-7.

26. Francobandiera G. Olanzapine augmentation of serotonin uptake inhibitors in obsessive-compulsive disorder: an open study. Can J Psychiatry. 2001 May;46(4):356-8.

27. Maina G, Pessina E, Albert U, Bogetto F. 8-week, single-blind, randomized trial comparing risperidone versus olanzapine augmentation of serotonin reuptake inhibitors in treatment-resistant obsessive-compulsive disorder. Eur Neuropsychopharmacol. 2008 May;18(5):364-72.

28. D'Amico G, Cedro C, Muscatello MR, Pandolfo G, Di Rosa AE, Zoccali R, et al. Olanzapine augmentation of paroxetine-refractory obsessive-compulsive disorder. Prog Neuropsychopharmacol Biol Psychiatry. 2003 Jun;27(4):619-23.

Cite this article as: Raichandani OP, Chaturvedi JN. Randomized, single blinded comparative study of selective serotonin reuptake inhibitor augmentation with Olanzapine v/s Amisulpride for obsessivecompulsive disorder. Int J Basic Clin Pharmacol 2019;8:1884-90. 